\title{
Machine Learning-based Prediction of Enzyme Substrate Scope: Application to Bacterial Nitrilases
}

\author{
Zhongyu Mou ${ }^{1}$, Jason Eakes ${ }^{1}$, Connor Cooper ${ }^{2}$, Carmen Foster ${ }^{1}$, Robert Standaert $^{1}$, \\ Mircea Podar ${ }^{1}$, Mitchel Doktycz ${ }^{1}$, and Jerry Parks ${ }^{1}$ \\ ${ }^{1}$ Oak Ridge National Laboratory \\ ${ }^{2}$ The University of Tennessee Knoxville-Oak Ridge National Laboratory Graduate School \\ of Genome Science and Technology
}

May 7, 2020

\begin{abstract}
Predicting the range of substrates accepted by an enzyme from its amino acid sequence is challenging. Although sequenceand structure-based annotation approaches are often accurate for predicting broad categories of substrate specificity, they generally cannot predict which specific molecules will be accepted as substrates for a given enzyme, particularly within a class of closely related molecules. Combining targeted experimental activity data with structural modeling, ligand docking, and physicochemical properties of proteins and ligands with various machine learning models provides complementary information that can lead to accurate predictions of substrate scope for related enzymes. Here we describe such an approach that can predict the substrate scope of bacterial nitrilases, which catalyze the hydrolysis of nitrile compounds to the corresponding carboxylic acids and ammonia. Each of the four machine learning models (linear regression, random forest, gradient-boosted decision trees, and support vector machines) performed similarly (average ROC $=0.9$, average accuracy $=\sim 82 \%$ ) for predicting substrate scope for this dataset. The approach is intended to be highly modular with respect to physicochemical property calculations and software used for docking and modeling.
\end{abstract}

\section{Hosted file}

nitrilases_submit_final.pdf available at https://authorea.com/users/319169/articles/448933machine-learning-based-prediction-of-enzyme-substrate-scope-application-to-bacterial-

nitrilases 